\title{
MicroRNA-1202 plays a vital role in osteoarthritis via KCNQ1OT1 has-miR-1202- ETS1 regulatory pathway
}

\author{
Changzeng Liu*, Jianming Gao, Guangyan Su, Yang Xiang and Le Wan
}

\begin{abstract}
Background: This study aimed to explore the molecular mechanism of osteoarthritis $(\mathrm{OA})$ and provide information about new genes as potential targets for OA treatment.

Methods: Gene expression profile of GSE105027, including 12 OA serum samples (OA group) and 12 healthy serum samples (ctrl group), was downloaded. The differentially expressed miRNAs (DEMs) as well as miRNA-mRNAs interactions were investigated, followed by function and pathway investigation. Then the protein-protein interaction (PPI) network was performed. Furthermore, the long non-coding RNA (IncRNA)-miRNA-mRNA interactions (competing endogenous RNAs, ceRNAs) were investigated.

Results: A total of 17 downregulated miRNAs were revealed between OA and ctrl groups. These DEMs such as hasmiR-1202 were mainly enriched in GO functions like histone acetyltransferase binding and KEGG pathways like cellular senescence. The integrated PPI network analysis showed that has-miR-1202, has-miR-33b-3p, has-miR-940, has-miR-4284, and has-miR-4281 were 5 downregulated miRNAs in this network. Furthermore, the IncRNA-miRNAmRNA interactions such as KCNQ1OT1-has-miR-1202-ETS1 were revealed in the present ceRNA network.

Conclusion: Key DEMs such as miR-33b-3p, miR-940, and miR-1202 may be involved in OA. miR-1202 may regulate OA development via histone acetyltransferase pathway binding function and cellular senescence pathway. Furthermore, KCNQ1OT1-has-miR-1202-ETS1 might be vital for the process of OA.
\end{abstract}

Keywords: Osteoarthritis, Differentially expressed miRNAs, Function and pathway analysis, Protein-protein interaction network, Competing endogenous RNAs

\section{Background}

Osteoarthritis (OA) is the wear-and-tear type of arthritis that causes joint pain and stiffness [1]. Worldwide, there are approximately 0.25 billion people suffering with OA [2]. The epidemiology of OA is very complex due to various factors such as genetic and biomechanical components [3], and the molecular mechanisms underlying OA are still not completely understood.

\footnotetext{
* Correspondence: liuchzeng88@163.com

Department of Orthopaedics, No. 904th Hospital of the Joint Logistics Support Force of PLA, Liangxi District, Wuxi 214044, Jiangsu, China
}

MicroRNAs (miRNAs) are commonly used to identify novel OA genes and their related inflammatory network [4]. A previous study shows that the upregulation of miR1 controls the development of OA via targeting FZD7 of Wnt $/ \beta$-catenin signaling [5]. Zhang et al. indicated that miR-320 might target matrix metalloproteinase-13 and further affected the process of OA [6]. Despite miRNAs, long non-coding RNAs (lncRNAs) are correlated with OA and are regulated by OA-associated factors [7, 8]. The previous study shows that lncRNAs take part in many kinds of pathological processes in OA such as extracellular matrix (ECM) [9]. A previous bioinformatics analysis

(c) The Author(s). 2020 Open Access This article is licensed under a Creative Commons Attribution 4.0 International License, which permits use, sharing, adaptation, distribution and reproduction in any medium or format, as long as you give appropriate credit to the original author(s) and the source, provide a link to the Creative Commons licence, and indicate if changes were made. The images or other third party material in this article are included in the article's Creative Commons licence, unless indicated otherwise in a credit line to the material. If material is not included in the article's Creative Commons licence and your intended use is not permitted by statutory regulation or exceeds the permitted use, you will need to obtain permission directly from the copyright holder. To view a copy of this licence, visit http://creativecommons.org/licenses/by/4.0/ The Creative Commons Public Domain Dedication waiver (http://creativecommons.org/publicdomain/zero/1.0/) applies to the data made available in this article, unless otherwise stated in a credit line to the data. 
indicates that lncRNAs (such as uc.343) and predicted target homeobox gene $\mathrm{C} 8$ (HOXC8) are promising therapeutic targets for OA [10]. Actually, the lncRNAsmiRNAs-mRNAs (competing endogenous RNAs, ceRNAs) regulatory network is an important tripartite axis in the regulation of the disease process [11, 12]. Although the identification of molecular factors contributes to the therapeutic interventions in OA [13], the key miRNAs and the possible regulation mechanism of ceRNA in OA are still unclear.

The bioinformatics analysis of gene expression profiles provides new opportunities to uncover the potential OA-related genes such as cathepsin $\mathrm{H}(\mathrm{CTSH})$ and cathepsin S (CTSS) [14-17]. In order to explore the potential differentially expressed miRNAs (DEMs) as a novel breakthrough in the clinical treatment of OA, Ntoumou et al. identified a circulating miRNA signature for OA patients using DEMs analysis and related pathway investigation [18]. They found that miRNAs such as miR140-3p regulated the metabolic processes of OA patients. However, the integrated regulatory mechanism involving DEMs, mRNAs, and lncRNAs on OA progression is still unclear. Based on the gene expression profile provided by Ntoumou et al., the present bioinformatics analysis revealed the DEMs between OA samples and control samples. Then, the miRNAs-mRNAs relations were explored, followed by the miRNAs-mRNAs regulatory network investigation. The protein-protein interaction (PPI) network was further constructed according to mRNAs in the miRNAs-mRNAs network. Finally, the ceRNA network was constructed and analyzed. The present study aimed to reveal the potential mechanism of $\mathrm{OA}$ and provide information about new genes as potential targets for OA treatment.

\section{Methods}

\section{Data resource and preprocessing}

Gene expression profile data in the dataset GSE105027 [18] were downloaded from Gene Expression Omnibus (GEO) database. This dataset was produced on GPL21575 Agilent-070156 Human_miRNA_V21.0_ Microarray 046064 platform. A total of 12 serum samples of OA patients (OA group) and 12 serum samples of healthy individuals (ctrl group) were included in the dataset.

The normalization for the downloaded original data was performed using the RAM75 [19, 20] method based on Affy package (version 1.50.0) in Rstudio software (version 1.1.414) [21]. The normalization process in this study included background correction, normalization, and expression quantification. If different probes targeted to the same miRNA (miRNA symbol), the mean value of different probes was considered as the final expression value of this miRNA.

\section{The investigation for DEMs}

The $P$ value and fold change (FC) of DEMs between the $\mathrm{OA}$ and ctrl group were calculated by Linear Models for Microarray Data (limma) package [22] in $\mathrm{R}$ (version 3.32.5) software. Then the $P$ value $<0.05$ and $\left|\log _{2} \mathrm{FC}\right|>$ 1 were selected as the thresholds for the identification of DEMs. The bidirectional hierarchical clustering for DEMs was then performed by pheatmap software (version 1.0.8).

\section{The miRNA-target gene investigation and network construction}

The predicted target genes of miRNAs were investigated using miRWalk 2.0 software (parameters, miRBase database; species, human) [23, 24]. The miRWalk [25], RNA22 [26], miRanda [27], and Targetscan [28] were selected as default databases for interaction scanning. The genes that appeared in all four databases were selected as target genes. Then, we downloaded the target genes of miRNAs which had been already verified in the validated target module of miRWalk 2.0 software. Based on overlapping results of predicted miRNA-target genes and verified miRNA-target genes, the miRNA-mRNAs interactions were finally explored. Furthermore, the miRNA-mRNAs interaction relations were visualized by Cytoscape (version 3.2.1, http://apps.cytoscape.org/) [29].

\section{Enrichment analysis of the DEGs}

The clusterProfiler software [30] is an online tool that provides enrichment analyses including Gene Ontology (GO) and Kyoto Encyclopedia of Genes and Genomes (KEGG). Based on the clusterProfiler software, the GO functional annotation and KEGG pathway enrichment analysis were performed on DEGs. $P$ value (the significance threshold of the hypergeometric test) $<0.05$ was chosen as the cutoff criterion for present enrichment analysis.

\section{Construction of PPI network}

The PPI research could reveal the interactions of proteins at the molecular level [31]. Search Tool for the Retrieval of Interacting Genes/Proteins (STRING, version 10.0) is a biological database of known and predicted PPI [32]. Using STRING database, PPI was constructed by the target genes that were regulated by miRNAs (required confidence (combined score) $>0.4$ ). Then, the integrated PPI network was further constructed based on the PPI network constructed by target genes regulated by miRNA and miRNA-target interactions, followed by visualization using Cytoscape software. The network topology analyses, including degree centrality (DC) [33], betweenness centrality (BC) [34], and closeness centrality (CC) [35], were performed by the CytoNCA [36] 
(version 2.1.6; parameter, without weight) to reveal the hub protein [19].

\section{Construction of ceRNA regulatory network}

The miRNA-associated IncRNAs were explored based on the $\operatorname{lnCeDB}$ database (http://www.lncrnablog.com/) [37]. The lncRNA-miRNA regulatory relations were constructed using Cytoscape software. Then, based on the lncRNA-miRNA relations and miRNA-DEMs interactions, the lncRNA-miRNA-mRNA (ceRNA) network was further explored. Finally, the ceRNA network was visualized by Cytoscape software.

\section{Results}

DEMs investigation between the $\mathrm{OA}$ group and ctrl group With $P$ value $<0.05$ and $\left|\log _{2} \mathrm{FC}\right|>1$, a total of 17 downregulated DEMs were obtained between the OA and ctrl groups. However, the result of the upregulated DEMs was negative. The bidirectional hierarchical clustering for DEMs is shown in Fig. 1. The results showed that the expression value of OA samples and ctrl samples could basically separate the two groups.

\section{The miRNAs-mRNAs interaction analysis}

A total of 5 downregulated miRNAs (has-miR-1202, hasmiR-33b-3p, has-miR-940, has-miR-4284, has-miR-4281) and 185 target mRNAs were screened by miRWalk 2.0 software. Then, the miRNAs-DEMs regulatory network (including a total of 190 nodes and 189 interactions) was constructed (Fig. 2).

\section{GO and KEGG analysis}

The results of the GO function and KEGG pathway enrichment analysis are listed in Tables 1 and 2, respectively. Hsa-miR-1202 was mainly involved in GO functions including histone acetyltransferase binding (GO:0035035; $P$ value, $1.28 \mathrm{E}-02$; gene, ETS protooncogene 1, transcription factor (ETS1)) and glutamate receptor activity (GO:0008066; $P$ value, 1.28E-02; gene, Rattus norvegicus glutamate receptor, metabotropic 4 (GRM4)). Meanwhile, has-miR-1202 was mainly enriched in pathways like cellular senescence (hsa05211; $P$ value, 4.29E-02; gene, ETS1) and taste transduction (hsa04742; $P$ value, 2.24E-02; gene, GRM4).

Hsa-miR-33b-3p was mainly involved in GO functions including motor activity (GO:0003774; $P$ value, 4.55E -03; gene, myosin IIIA $(M Y O 3 A)$ ) and microfilament motor activity (GO:0000146; $P$ value, 1.70E-02; gene, MYO3A). Meanwhile, hsa-miR-33b-3p was mainly enriched in KEGG pathways like collecting duct acid secretion (hsa04966; $P$ value, 2.53E-02; gene, solute carrier family 12 member 7 (SLC12A7)) and circadian rhythm (has04710; $P$ value, 2.90E-02; gene, retinoid-related orphan receptor alpha $(R O R A))$.

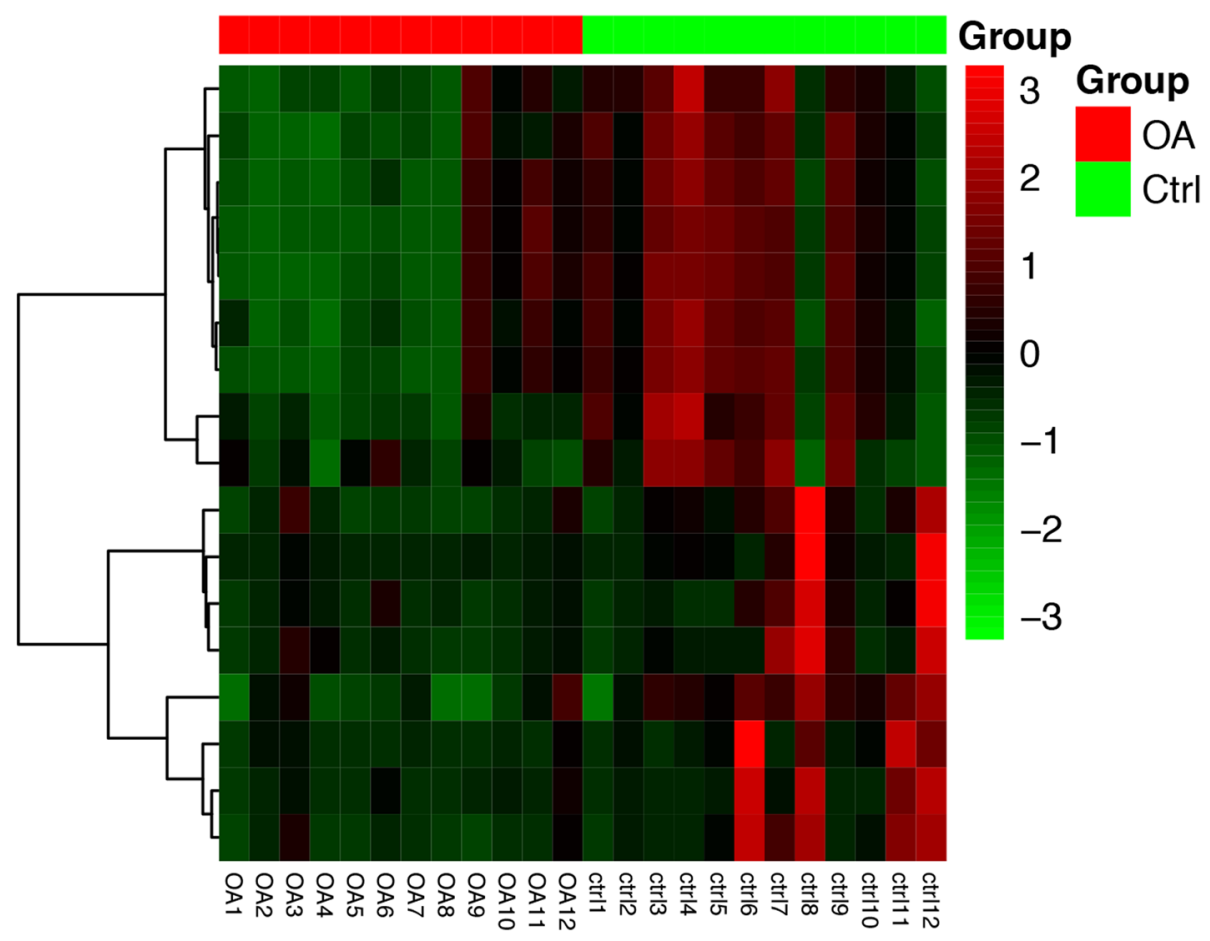

Fig. 1 The heat map for differentially expressed miRNAs between the osteoarthritis group and control group. The red block represents the genes in the osteoarthritis group; the green block represents the genes in the control group; and the red color represents upregulation, while green color represents downregulation 


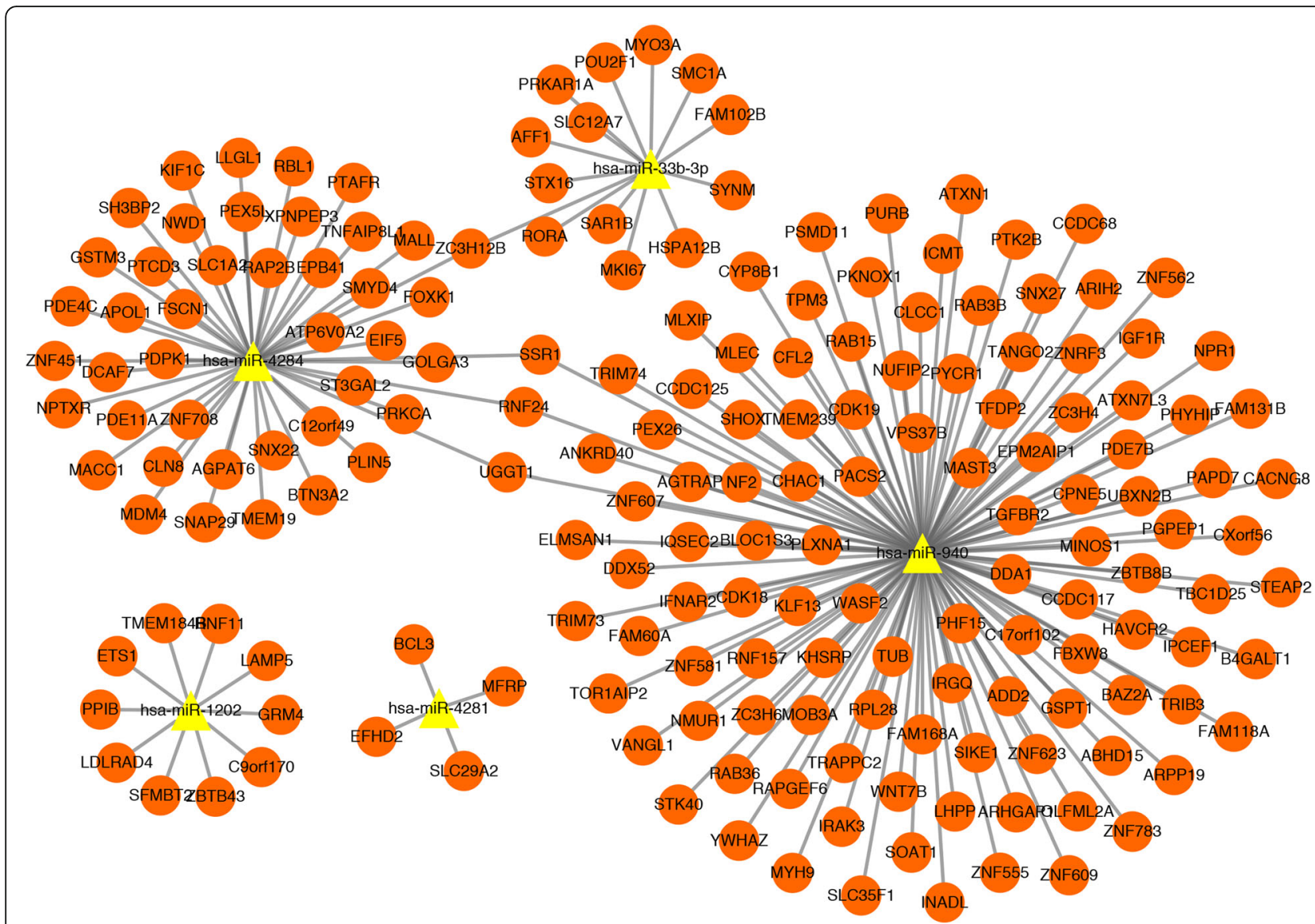

Fig. 2 The miRNAs-mRNAs regulatory network. The orange dot represents mRNA, and the yellow triangle represents the downregulated miRNA

Hsa-miR-4281 was mainly involved in GO functions including nucleoside transmembrane transporter activity (GO:0005337; $P$ value, 3.10E-03; gene, solute carrier family 29 member 2 (SLC29A2)) and nucleobasecontaining compound transmembrane transporter activity (GO:0015932; $P$ value, 6.91E-03; gene, SLC29A2). Meanwhile, hsa-miR-4281 was mainly enriched in KEGG pathways like TNF signaling pathway (hsa04668; $P$ value, 1.46E-02; gene, B cell CLL/lymphoma 3 (BCL3)).

Hsa-miR-4284 was mainly involved in GO functions including 3',5'-cyclic-nucleotide phosphodiesterase activity (GO:0004114; $P$ value, 1.64E-03; gene, phosphodiesterase $4 \mathrm{C} \quad(P D E 4 C))$ and cyclic-nucleotide phosphodiesterase activity (GO:0004112; $P$ value, $1.78 \mathrm{E}$ -03; gene, PDE4C). Meanwhile, hsa-miR-4284 was mainly enriched in pathways like morphine addiction (hsa05032; $P$ value, 2.05E-03; gene, protein kinase $C$ alpha (PRKCA)) and African trypanosomiasis (hsa05143; $P$ value, 4.33E-03; gene, $P R K C A)$.

\section{PPI network analysis}

The PPI network was constructed with the target genes of DEMs using STRING database. With a combined score of $>0.4$, a total of 68 interaction relations and 75 nodes were identified in the PPI network. Based on the topology analysis, the top 5 hub proteins (according to the degree) were PRKCA (DC, 6.0; $\mathrm{BC}, 743.0 ; \mathrm{CC}$, 0.02647585), InaD-like protein (INADL) (DC, 5.0; BC, 319.0; CC, 0.026176158), cAMP-dependent protein kinase type I-alpha regulatory subunit (PRKAR1A) (DC, 5.0; BC, 333.0; CC, 0.026111504), myosin heavy chain type II isoform A (MYH9) (DC, 5.0; BC, 309.0; CC, $0.026222536)$ and natriuretic peptide receptor $\mathrm{A} /$ guanylate cyclase A (NPR1) (DC, 5.0; BC, 77.0; CC, 0.025819957). The integrated PPI network included 190 nodes, 257 relations, and 5 downregulated miRNAs (including has-miR-33b-3p, has-miR-1202, has-miR-4281, has-miR-4284, and has-miR-940) (Fig. 3).

\section{ceRNA regulatory network investigation}

The $\ln C e D B$ database was used to explore the lncRNAs related with 5 miRNAs mentioned above. The results showed that a total of 172 lncRNAs and 215 interactions were investigated in miRNAslncRNAs regulatory relations. With the binding-site number $>2$, a lncRNA-miRNA regulatory network 
Table 1 The results of GO function analysis for downregulated miRNAs

\begin{tabular}{|c|c|c|c|c|}
\hline Cluster & $\mathrm{GO}$ & $\boldsymbol{P}$ value & Count & Symbol \\
\hline hsa-miR-1202 & GO:0070412 R-SMAD binding & 1.09E-02 & 1 & LDLRAD4 \\
\hline hsa-miR-1202 & GO:0008066 glutamate receptor activity & $1.28 \mathrm{E}-02$ & 1 & GRM4 \\
\hline hsa-miR-1202 & GO:0035035 histone acetyltransferase binding & $1.28 \mathrm{E}-02$ & 1 & ETS1 \\
\hline hsa-miR-1202 & GO:0070063 RNA polymerase binding & $1.75 E-02$ & 1 & PPIB \\
\hline hsa-miR-1202 & GO:0003755 peptidyl-prolyl cis-trans isomerase activity & $2.04 \mathrm{E}-02$ & 1 & PPIB \\
\hline hsa-miR-1202 & GO:0016859 cis-trans isomerase activity & 2.13E-02 & 1 & PPIB \\
\hline hsa-miR-1202 & GO:0005518 collagen binding & $3.16 \mathrm{E}-02$ & 1 & PPIB \\
\hline hsa-miR-1202 & GO:0046332 SMAD binding & $3.30 \mathrm{E}-02$ & 1 & LDLRAD4 \\
\hline hsa-miR-1202 & GO:0051082 unfolded protein binding & 4.73E-02 & 1 & PPIB \\
\hline hsa-miR-33b-3p & GO:0003774 motor activity & 4.55E-03 & 2 & MYO3A/SMC1A \\
\hline hsa-miR-33b-3p & GO:0019215 intermediate filament binding & $1.01 \mathrm{E}-02$ & 1 & SYNM \\
\hline hsa-miR-33b-3p & GO:0030898 actin-dependent ATPase activity & $1.01 \mathrm{E}-02$ & 1 & MYO3A \\
\hline hsa-miR-33b-3p & GO:0034236 protein kinase A catalytic subunit binding & 1.16E-02 & 1 & PRKAR1A \\
\hline hsa-miR-33b-3p & GO:0000146 microfilament motor activity & 1.70E-02 & 1 & MYO3A \\
\hline hsa-miR-33b-3p & GO:0001221 transcription cofactor binding & $1.70 \mathrm{E}-02$ & 1 & RORA \\
\hline hsa-miR-33b-3p & GO:0030552 cAMP binding & 1.85E-02 & 1 & PRKAR1A \\
\hline hsa-miR-33b-3p & GO:0030291 protein serine/threonine kinase inhibitor activity & 2.23E-02 & 1 & PRKAR1A \\
\hline hsa-miR-33b-3p & GO:0043531 ADP binding & $2.53 \mathrm{E}-02$ & 1 & MYO3A \\
\hline hsa-miR-33b-3p & GO:0030551 cyclic nucleotide binding & $2.76 \mathrm{E}-02$ & 1 & PRKAR1A \\
\hline hsa-miR-33b-3p & GO:0005484 SNAP receptor activity & $2.91 \mathrm{E}-02$ & 1 & STX16 \\
\hline hsa-miR-33b-3p & GO:0008307 structural constituent of muscle & 3.06E-02 & 1 & SYNM \\
\hline hsa-miR-33b-3p & GO:0051018 protein kinase A binding & $3.21 \mathrm{E}-02$ & 1 & PRKAR1A \\
\hline hsa-miR-33b-3p & GO:0032934 sterol binding & $3.29 \mathrm{E}-02$ & 1 & RORA \\
\hline hsa-miR-33b-3p & $\begin{array}{l}\text { GO:0004879 RNA polymerase II transcription factor activity, } \\
\text { ligand-activated sequence-specific DNA binding }\end{array}$ & $3.74 \mathrm{E}-02$ & 1 & RORA \\
\hline hsa-miR-33b-3p & GO:0015296 anion:cation symporter activity & $3.74 \mathrm{E}-02$ & 1 & SLC12A7 \\
\hline hsa-miR-33b-3p & $\begin{array}{l}\text { GO:0098531 transcription factor activity, direct ligand regulated } \\
\text { sequence-specific DNA binding }\end{array}$ & $3.74 \mathrm{E}-02$ & 1 & RORA \\
\hline hsa-miR-33b-3p & GO:0003707 steroid hormone receptor activity & $4.41 \mathrm{E}-02$ & 1 & RORA \\
\hline hsa-miR-33b-3p & GO:0003682 chromatin binding & 4.85E-02 & 2 & POU2F1/SMC1A \\
\hline hsa-miR-4281 & $\begin{array}{l}\text { GO:0005337 nucleoside transmembrane transporter } \\
\text { activity }\end{array}$ & $3.10 \mathrm{E}-03$ & 1 & SLC29A2 \\
\hline hsa-miR-4281 & $\begin{array}{l}\text { GO:0015932 nucleobase-containing compound } \\
\text { transmembrane transporter activity }\end{array}$ & $6.91 \mathrm{E}-03$ & 1 & SLC29A2 \\
\hline hsa-miR-4281 & GO:1901505 carbohydrate derivative transporter activity & 7.15E-03 & 1 & SLC29A2 \\
\hline hsa-miR-4281 & GO:0030674 protein binding, bridging & 3.63E-02 & 1 & $\mathrm{BCL} 3$ \\
\hline hsa-miR-4281 & GO:0060090 binding, bridging & 4.00E-02 & 1 & $\mathrm{BCL} 3$ \\
\hline hsa-miR-4284 & GO:0004114 3',5'-cyclic-nucleotide phosphodiesterase activity & 1.64E-03 & 2 & PDE4C/PDE11A \\
\hline hsa-miR-4284 & GO:0004112 cyclic-nucleotide phosphodiesterase activity & $1.78 \mathrm{E}-03$ & 2 & PDE4C/PDE11A \\
\hline
\end{tabular}

GO Gene Ontology; $P<0.05$ was considered to be significantly different

(including 3 miRNAs and 30 lncRNAs) was constructed using Cytoscape software. Then, based on the IncRNA-miRNA relations and miRNA-DEMs interactions, the IncRNA-miRNA-mRNA interactions (ceRNA) including KCNQ1 overlapping transcript 1 (KCNQ1OT1)-has-miR-1202-ETS1 was further explored. Finally, the ceRNA network was visualized by Cytoscape software (Fig. 4).

\section{Discussion}

$\mathrm{OA}$ is the most common form of joint disease [38]. miRNAs are expressed in a different fashion in osteoarthritic 
Table 2 The results of KEGG pathway enrichment analysis for down-regulated miRNAs

\begin{tabular}{lllll}
\hline Cluster & Pathway & $\boldsymbol{P}$ value & Count & Gene \\
\hline hsa-miR-1202 & (hsa05211) Renal cell carcinoma & $1.86 \mathrm{E}-02$ & 1 & ETS1 \\
hsa-miR-1202 & (hsa04742) Taste transduction & $2.24 \mathrm{E}-02$ & 1 & GRM4 \\
hsa-miR-1202 & (hsa04724) Glutamatergic synapse & $3.06 \mathrm{E}-02$ & 1 & GRM4 \\
hsa-miR-1202 & (hsa04072) Phospholipase D signaling pathway & $3.92 \mathrm{E}-02$ & 1 & GRM4 \\
hsa-miR-1202 & (hsa04218) Cellular senescence & $4.29 \mathrm{E}-02$ & 1 & ETS1 \\
hsa-miR-33b-3p & (hsa04966) Collecting duct acid secretion & $2.53 \mathrm{E}-02$ & 1 & SLC12A7 \\
hsa-miR-33b-3p & (hsa04710) Circadian rhythm & $2.90 \mathrm{E}-02$ & 1 & RORA \\
hsa-miR-33b-3p & (hsa04130) SNARE interactions in vesicular transport & $3.18 \mathrm{E}-02$ & 1 & STX16 \\
hsa-miR-4281 & (hsa04668) TNF signaling pathway & $1.46 E-02$ & 1 & BCL3 \\
hsa-miR-4284 & (hsa05032) Morphine addiction & $2.05 E-03$ & 3 & PDE4C/PDE11A/PRKCA \\
hsa-miR-4284 & (hsa05143) African trypanosomiasis & $4.33 E-03$ & 2 & APOL1/PRKCA \\
hsa-miR-4284 & (hsa04960) Aldosterone-regulated sodium reabsorption & $4.83 E-03$ & 2 & PDPK1/PRKCA \\
\hline
\end{tabular}

KEGG Kyoto Encyclopedia of Genes and Genomes; $P<0.05$ was considered to be significantly different.

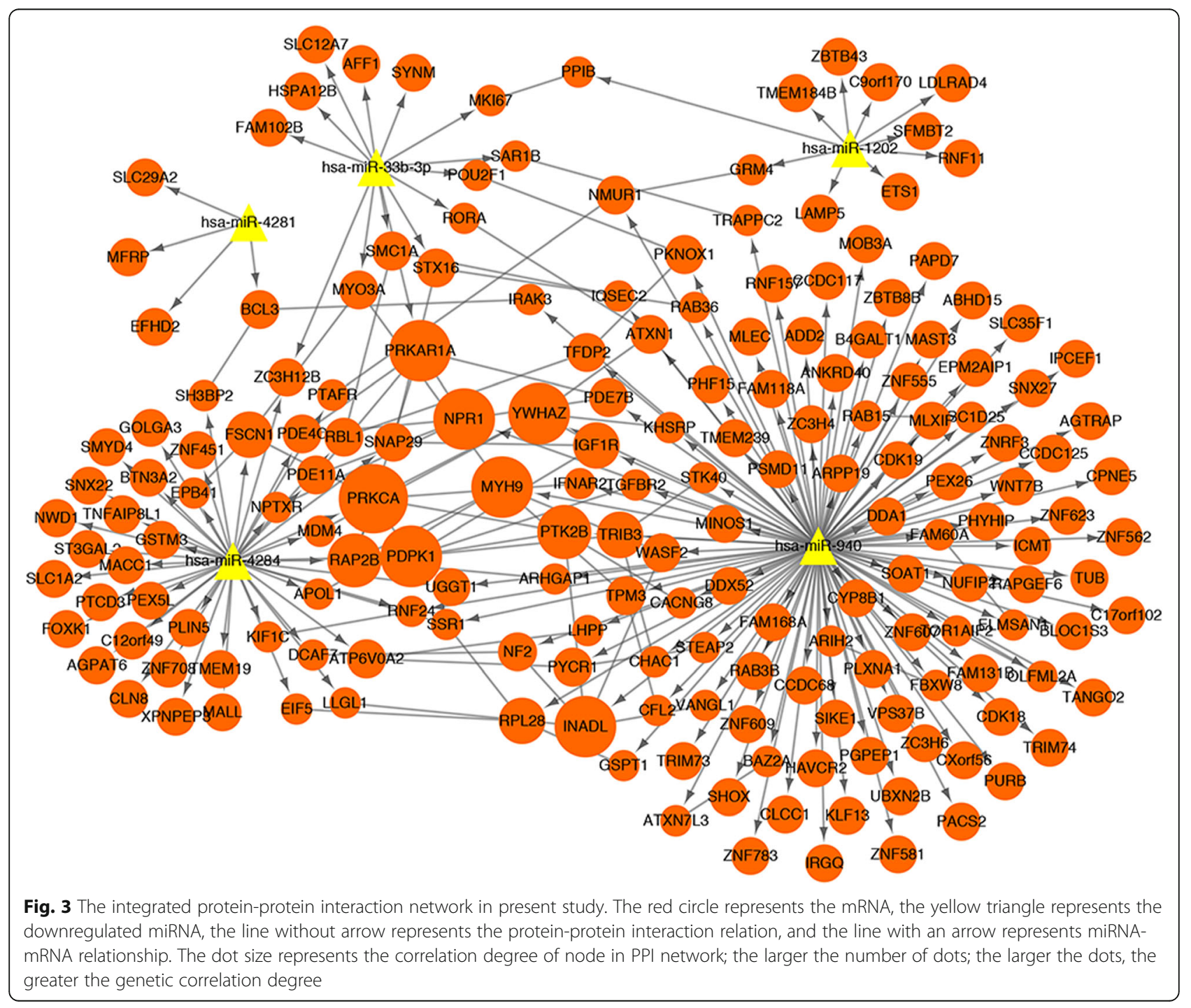




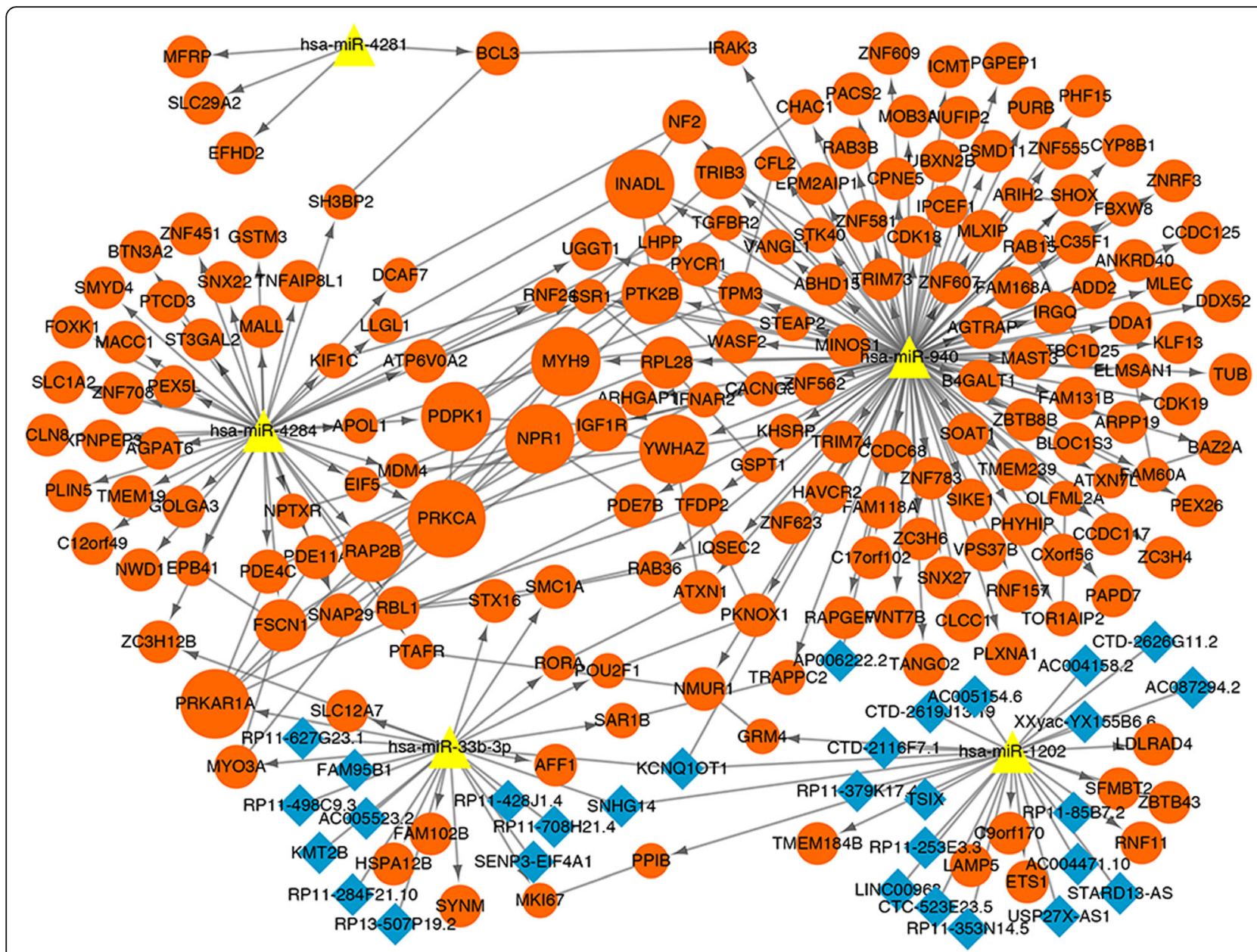

Fig. 4 The ceRNA network in this study. The orange dot represents mRNA; the blue diamond represents IncRNA; and the yellow triangle represents the downregulated miRNA. The dot size represents the correlation degree of node in PPI network; the larger the dots, the greater the genetic correlation degree

relative to nonosteoarthritic cartilage and may be useful for diagnosis or management of OA [33, 34]. In the present study, a total of 17 downregulated miRNAs were revealed between the OA group and ctrl group. These DEMs such as has-miR-1202 were mainly enriched in GO functions like histone acetyltransferase binding and KEGG pathways like cellular senescence. The integrated PPI network analysis showed that has-miR-1202, hasmiR-33b-3p, has-miR-940, has-miR-4284, and has-miR4281 were 5 downregulated miRNAs in this network. Furthermore, the IncRNA-miRNA-mRNA interactions such as KCNQ1OT1-has-miR-1202-ETS1 were revealed in the present ceRNA network.

miRNAs are increasingly implicated in the pathogenesis of complex diseases such as cancer and OA [35, 39]. Based on bioinformatics and proteomic analysis, Dimitrios et al. indicated that the collaborative networks of miRNAs and proteins were associated with OA pathogenesis [40]. In previous studies, serum miRNA array analysis showed that miR-33b-3p may be used as a potential biomarker for OA [41]. Meanwhile, miR-940 is involved in the inflammatory response of chondrocytes in OA [42]. In this study, miR-33b-3p and miR-940 were downregulated in the OA group, suggesting the potential role of them in OA. As a member of the miRNA family, miR-1202 suppresses proliferation and induces endoplasmic reticulum stress in tumor cells, which suggest a potential therapy of miR-1202 in clinical treatment [43]. Despite that, miR-1202 is a primate-specific and brainenriched miRNA [44]. However, the function of miR1202 in OA is still unclear. In this study, miR-1202 was an outstanding downregulated miRNA between the OA group and ctrl group, suggesting that miR-1202 might take part in the progression of OA.

In addition, complex interactions existed between miRNAs and their multiple target genes, which may be important in gene regulation and the control of homeostatic pathways in OA [33, 34]. As a target gene of miR-1202, 
ETS1 is a member of the ETS family of transcription factors [45]. ETS1 has been proved to associate with regulation of immune cell function and with an aggressive behavior in tumors [46]. Xu et al. indicated that miR-221-3p modulated ETS1 expression in synovial fibroblasts of OA patients [47], suggesting the potential role of ETS1 in OA. Histone acetylation has a close relation with some biological effects such as gene transcription and is catalyzed by histone acetyltransferases [48]. A previous study shows that ETS1 synergistically activates the gene expression of guanylyl cyclase/ natriuretic peptide receptor A via histone acetyltransferase pathway [49]. However, the detailed mechanism of ETS1 and histone acetyltransferase pathway in $\mathrm{OA}$ is unclear. Moreover, ETS1 can activate the p16INK4a promoter through an ETS-binding site via cellular senescence function [50]. In this study, miR-1202 and ETS1 were enriched in histone acetyltransferase binding function and cellular senescence pathway. Thus, based on the present study, we speculated that the downregulated miR-1202 might target EST1 and further affect the OA progression via histone acetyltransferase pathway binding and cellular senescence pathway.

LncRNAs are proved to participate in a variety of biological processes as regulatory molecules [51]. The expression of lncRNAs has recently been reported in tumorigenesis and plays a pivotal role in regulating behavior cell cycle [52]. A previous study shows that lncRNA functions as a ceRNA to promote cartilage degradation in human OA [8]. KCNQ1OT1 is a nuclear transcript found in close proximity to the nucleolus in certain cell types [53]. It involves in certain imprinted gene network that may play a role in various diseases including Beckwith-Wiedemann syndrome and coronary artery lesion [54, 55]. However, the effect of KCNQ1OT1 on OA is still unclear. Our ceRNA network analysis showed that KCNQ1OT1-has-miR-1202-ETS1 was one of the most outstanding ceRNA. Thus, we speculated that the IncRNA KCNQ1OT1 might play an important role in OA progression via sponging has-miR1202-ETS1 interaction.

However, there were some limitations in this study such as small sample size and lack of verification test, which will introduce false-positive results and consequently influence the reliability of our findings. Thus, a large sample size with a wide verification analysis is needed in further investigation.

In conclusion, the present study showed that downregulated DEMs such as miR-33b-3p, miR-940, and miR-1202 may be involved in OA. miR-1202 might target EST1 to regulate the activation of histone acetyltransferase pathway binding and cellular senescence pathway and thus further affect OA development. Furthermore, KCNQ1OT1-hasmiR-1202-ETS1 might play an important role in the process of OA.

\section{Acknowledgements}

None.

\section{Authors' contributions}

Conception and design of the research: CL; acquisition of data: GS and JG; analysis and interpretation of data: YX; statistical analysis: LW; drafting the manuscript: CL; revision of manuscript for important intellectual content: GS. The authors read and approved the final manuscript.

Funding

None.

Availability of data and materials

The datasets used and analyzed during the current study are available from the corresponding author on reasonable request.

Ethics approval and consent to participate

Not applicable.

Consent for publication

Not applicable.

\section{Competing interests}

The authors declare that they have no conflict of interest.

Received: 3 March 2020 Accepted: 27 March 2020

Published online: 06 April 2020

\section{References}

1. Glyn-Jones S, Palmer AJ, Agricola R, Price AJ, Vincent TL, Weinans $H$, et al. Osteoarthritis. Lancet. 2015. https://doi.org/10.1016/S0140-6736(14)60802-3.

2. Vos T, Flaxman AD, Naghavi M, Lozano R, Michaud C, Ezzati M, et al. Years lived with disability (YLDs) for 1160 sequelae of 289 diseases and injuries 1990-2010: a systematic analysis for the Global Burden of Disease Study 2010. Lancet. 2012;380(9859):2163-96. https://doi.org/10.1016/S01406736(12)61729-2.

3. Johnson VL, Hunter DJ. The epidemiology of osteoarthritis. Best Pract Res Clin Rheumatol. 2014;28(1):5-15. https://doi.org/10.1016/j.berh.2014.01.004

4. Iliopoulos D, Malizos KN, Oikonomou P, Tsezou A. Integrative microRNA and proteomic approaches identify novel osteoarthritis genes and their collaborative metabolic and inflammatory networks. Plos One. 2007;3(11): e3740.

5. Xing D, Wang B, Xu Y, Tao K, Lin J. Overexpression of microRNA-1 controls the development of osteoarthritis via targeting FZD7 of Wnt/ $\beta$-catenin signaling. Osteoarthritis Cartilage. 2016;24:S181-S2

6. Zhang Z, Meng F, Huang G, Kang Y, Sheng P, Liao W. Regulation of matrix metalloproteinase-13 by MIRNA-320 in chondrogenesis and osteoarthritis. Osteoarthritis Cartilage. 2016;24:S381-S.

7. Katsara O, Attur M, Kolupaeva V. Targeting a selective pool of mRNAs to interfere with progression of osteoarthritis (OA). Osteoarthritis Cartilage. 2017:25(1):S149.

8. Liu Q, Hu X, Zhang X, Dai L, Duan X, Zhou C, et al. The TMSB4 pseudogene LncRNA functions as a competing endogenous RNA to promote cartilage degradation in human osteoarthritis. Mol Ther. 2016;24(10):1726-33.

9. Chen WK, Yu XH, Wei Y, Cheng W, He WS, Yan YG, et al. IncRNAs: novel players in intervertebral disc degeneration and osteoarthritis. Cell Prolif. 2017:50(1).

10. Huang G, Fu M, Zhang Z, Liu J, Huang Z, Wu P, et al. Expression profile of long noncoding rnas in osteoarthritis patients. Osteoarthritis Cartilage. 2014; 22(4):S230-S.

11. Wu C, Arora P. Long noncoding RNA-microRNA-mRNA: a novel tripartite axis in the regulation of cardiac hypertrophy. Circ Cardiovasc Genet. 2014; 7(5):729-31.

12. Ye $S$, Yang $L$, Zhao $X$, Song $W$, Wang $W$, Zheng S. Bioinformatics method to predict two regulation mechanism: TF-miRNA-mRNA and IncRNA-miRNAmRNA in pancreatic cancer. Cell Biochem Biophys. 2014;70(3):1849-58.

13. Aigner T, Fundel K, Saas J, Gebhard PM, Haag J, Weiss T, et al. Large-scale gene expression profiling reveals major pathogenetic pathways of cartilage degeneration in osteoarthritis. Arthritis Rheum. 2006;54(11):3533-44. https:// doi.org/10.1002/art.22174. 
14. $\mathrm{Ma}$ CH, Lv Q, Cao Y, Wang Q, Zhou XK, Ye BW, et al. Genes relevant with osteoarthritis by comparison gene expression profiles of synovial membrane of osteoarthritis patients at different stages. Eur Rev Med Pharmacol Sci. 2014;18(3):431-9.

15. Lambert C, Dubuc JE, Montell E, Verges J, Munaut C, Noel A, et al. Gene expression pattern of cells from inflamed and normal areas of osteoarthritis synovial membrane. Arthritis Rheumatol. 2014;66(4):960-8. https://doi.org/ 10.1002/art.38315.

16. Wang W, Yang L, Hao J, Zheng S, Yan W, Xiao X, et al. Comparative analysis of gene expression profiles of hip articular cartilage between non-traumatic necrosis and osteoarthritis. Gene. 2016;591(1):43-7.

17. Park R, Ji JD. Unique gene expression profile in osteoarthritis synovium compared with cartilage: analysis of publicly accessible microarray datasets. Rheumatol Int. 2016;75(Suppl 1):1-9.

18. Ntoumou E, Tzetis M, Braoudaki M, Lambrou G, Poulou M, Malizos K, et al. Serum microRNA array analysis identifies miR-140-3p, miR-33b-3p and miR671-3p as potential osteoarthritis biomarkers involved in metabolic processes. Clin Epigenet. 2017;9(1):127.

19. Bolstad BM, Irizarry RA, Astrand M, Speed TP. A comparison of normalization methods for high density oligonucleotide array data based on variance and bias. Bioinformatics. 2003;19(2):185-93.

20. Irizarry RA, Hobbs B, Collin F, Beazer-Barclay YD, Antonellis K, Scherf U, et al. Exploration, normalization, and summaries of high density oligonucleotide array probe level data. Biostatistics. 2003;4(2):249-64.

21. Gautier L, Cope L, Bolstad BM, Irizarry RA. affy —analysis of Affymetrix GeneChip data at the probe level. Bioinformatics. 2004;20(3):307-15.

22. Smyth GK. Limma: linear models for microarray data, in Bioinformatics and computational biology solutions using R and Bioconductor: Springer; 2005. p. 397-420.

23. Breuer K, Foroushani AK, Laird MR, Chen C, Sribnaia A, Lo R, et al. InnateDB: systems biology of innate immunity and beyond--recent updates and continuing curation. Nucleic Acids Res. 2013;41(Database issue):D1228-33. https://doi.org/10.1093/nar/gks1147.

24. Dweep H, Gretz N. miRWalk2. 0: a comprehensive atlas of microRNA-target interactions. Nat Methods. 2015;12(8):697.

25. Dweep H, Gretz N, Sticht C. miRWalk database for miRNA-target interactions. Methods Mol Biol. 2014;1182:289-305.

26. Rigoutsos I, Miranda K, Huynh T. rna22: A unified computational framework for discovering miRNA precursors, localizing mature miRNAs, identifying $3^{\prime}$ UTR target-islands, and determining the targets of mature-miRNAs. Ibm Corporation. 2007.

27. Betel D, Koppal A, Agius P, Sander C, Leslie C. Comprehensive modeling of microRNA targets predicts functional non-conserved and non-canonical sites. Genome Biol. 2010;11(8):R90-R.

28. Agarwal V, Bell GW, Nam JW, Bartel DP. Predicting effective microRNA target sites in mammalian mRNAs. Elife Sci. 2015;4:e05005.

29. Shannon P, Markiel A, Ozier O, Baliga NS, Wang JT, Ramage D, et al. Cytoscape: a software environment for integrated models of biomolecular interaction networks. Genome Res. 2003;13(11):2498-504.

30. Yu G, Wang $L G$, Han $Y$, He QY. clusterProfiler: an R package for comparing biological themes among gene clusters. Omics J Integ Biol. 2012;16(5):284-7.

31. Giot L, Bader JS, Brouwer C, Chaudhuri A, Kuang B, Li Y, et al. A protein interaction map of Drosophila melanogaster. Science. 2003;302(5651):172736. https://doi.org/10.1126/science.1090289.

32. Szklarczyk D, Franceschini A, Wyder S, Forslund K, Heller D, Huerta-Cepas J, et al. STRING v10: protein-protein interaction networks, integrated over the tree of life. Nucleic Acids Res. 2015;43(Database issue):D447.

33. Oliviero A, Della Porta G, Peretti GM, Maffulli N. MicroRNA in osteoarthritis: physiopathology, diagnosis and therapeutic challenge. Br Med Bull. 2019; 130(1):137-47.

34. Oliviero A, Della Porta G, Peretti GM, Maffulli N. Corrigendum to: MicroRNA in osteoarthritis: physiopathology, diagnosis and therapeutic challenge. $\mathrm{Br}$ Med Bull. 2019;28(10).

35. Mraz M, Pospisilova S. MicroRNAs in chronic lymphocytic leukemia: from causality to associations and back. Expert Rev Hematol. 2012;5(6):579-81.

36. Tang Y, Li M, Wang J, Pan Y, Wu FX. CytoNCA: a cytoscape plugin for centrality analysis and evaluation of protein interaction networks. Biosystems. 2015;127:67-72. https://doi.org/10.1016/j.biosystems.2014.11.005.

37. Das S, Ghosal S, Sen R, Chakrabarti J. InCeDB: database of human long noncoding RNA acting as competing endogenous RNA. Plos One. 2014;9(6): e98965.
38. Zhang M, Wang J. Epigenetics and osteoarthritis. Genes Dis. 2015;2(1):69-75. https://doi.org/10.1016/i.gendis.2014.12.005.

39. Miyaki S, Asahara H. Macro view of microRNA function in osteoarthritis. Nat Rev Rheumatol. 2012:8(9):543.

40. Dimitrios I, Malizos KN, Pagona O, Aspasia T. Integrative microRNA and proteomic approaches identify novel osteoarthritis genes and their collaborative metabolic and inflammatory networks. Plos One. 2008;3(11):e3740.

41. Ntoumou E, Tzetis M, Braoudaki M, Lambrou G, Poulou M, Malizos K, et al. Serum microRNA array analysis identifies miR-140-3p, miR-33b-3p and miR$671-3 p$ as potential osteoarthritis biomarkers involved in metabolic processes. Clin Epigenetics. 2017:9(127):017-0428.

42. Cao J, Liu Z, Zhang L, Li J. miR-940 regulates the inflammatory response of chondrocytes by targeting MyD88 in osteoarthritis. Mol Cell Biochem. 2019; 461 (1-2):183-93.

43. Quan Y, Song Q, Wang J, Zhao L, LV J, Gong S. MiR-1202 functions as a tumor suppressor in glioma cells by targeting Rab1A. Tumor Biology. 2017; 39(4):101042831769756.

44. Lopez JP, Lim R, Cruceanu C, Crapper L, Fasano C, Labonte B, et al. miR1202 is a primate-specific and brain-enriched microRNA involved in major depression and antidepressant treatment. Nat Med. 2014;20(7):764-8.

45. Dittmer J. The biology of the ETS1 proto-oncogene. Mol Cancer. 2003;2(1):29.

46. Garrett-Sinha LA. Review of Ets1 structure, function, and roles in immunity. Cell Mol Life Sci. 2013;70(18):3375-90.

47. Xu J, Liu Y, Deng M, Li J, Cai H, Meng Q, et al. MicroRNA221-3p modulates ETS-1 expression in synovial fibroblasts from patients with osteoarthritis of temporomandibular joint. Osteoarthritis Cartilage. 2016;24(11):2003.

48. Chen J, Zhang X. Progress in the research of histone acetyltransferases. Chin Bull Lifeences. 2000;12.

49. Kumar P, Pandey KN. Histone acetyltransferase p300 and ETS-1 synergistically activate guanylyl cyclase/natriuretic peptide receptor-A gene expression. FASEB J. 2009;23.

50. Ohtani N, Zebedee Z, Huot TJG, Stinson JA, Sugimoto M, Ohashi Y, et al. Opposing effects of ETS and Id proteins on p16INK4a expression during cellular senescence. Nature. 2001;409(6823):1067-70.

51. Saakshi J, Deeksha B, Kumar LM, Sridhar S, Vinod S. Systematic transcriptome wide analysis of IncRNA-miRNA interactions. Plos One. 2013; 8(2):e53823.

52. Teng H, Wang P, Xue Y, Liu X, Ma J, Cai H, et al. Role of HCP5-miR-139RUNX1 feedback loop in regulating malignant behavior of glioma cells. Mol Ther J Am Soc Gene Ther. 2016;24(10):1806

53. Fedoriw AM, Calabrese JM, Mu W, Yee D, Magnuson T. Differentiationdriven nucleolar association of the mouse imprinted Kcnq1 locus. G3 Genes Genet. 2012;2(12):1521-8.

54. Arima T, Kamikihara T, Hayashida T, Kato K, Inoue T, Shirayoshi Y, et al. ZAC, LIT1 (KCNQ1OT1) and p57KIP2 (CDKN1C) are in an imprinted gene network that may play a role in Beckwith-Wiedemann syndrome. 2005:33(8):2650-60

55. Han HK, Chuan HE, Min-Sheng AN, Jin JF, Zhang CW, Chen QJ, et al. Correlation study between serum level of LncR NA KCNQ1OT1 overlapping transcript and coronary artery lesion in relevant patients. Chin Circ J. 2017.

\section{Publisher's Note}

Springer Nature remains neutral with regard to jurisdictional claims in published maps and institutional affiliations.

Ready to submit your research? Choose BMC and benefit from:

- fast, convenient online submission

- thorough peer review by experienced researchers in your field

- rapid publication on acceptance

- support for research data, including large and complex data types

- gold Open Access which fosters wider collaboration and increased citations

- maximum visibility for your research: over $100 \mathrm{M}$ website views per year

At BMC, research is always in progress.

Learn more biomedcentral.com/submissions 